\title{
AIGaN Nanostructures with Extremely High Room-Temperature Internal Quantum Efficiency of Emission Below 300 nm
}

\author{
A.A. TOROPOV, ${ }^{1,4}$ E.A. SHEVCHENKO,${ }^{1}$ T.V. SHUBINA, ${ }^{1}$ V.N. JMERIK, ${ }^{1}$ \\ D.V. NECHAEV ${ }^{1}$ E.A. EVROPEYTSEV ${ }^{1}$ V. KH. KAIBYSHEV ${ }^{1}$ G. POZINA, ${ }^{2}$ \\ S. ROUVIMOV, ${ }^{3}$ and S.V. IVANOV ${ }^{1}$ \\ 1.-Ioffe Institute, Politekhnicheskaya 26, St.Petersburg, Russia 194021. 2.-Department of \\ Physics, Chemistry and Biology, Linköping University, 58183 Linköping, Sweden. 3.-University \\ of Notre Dame, Notre Dame, IN 46556, USA. 4.—e-mail: toropov@beam.ioffe.ru
}

We present theoretical optimization of the design of a quantum well (QW) heterostructure based on AlGaN alloys, aimed at achievement of the maximum possible internal quantum efficiency of emission in the mid-ultraviolet spectral range below $300 \mathrm{~nm}$ at room temperature. A sample with optimized parameters was fabricated by plasma-assisted molecular beam epitaxy using the submonolayer digital alloying technique for QW formation. High-angle annular dark-field scanning transmission electron microscopy confirmed strong compositional disordering of the thus-fabricated QW, which presumably facilitates lateral localization of charge carriers in the QW plane. Stress evolution in the heterostructure was monitored in real time during growth using a multibeam optical stress sensor intended for measurements of substrate curvature. Time-resolved photoluminescence spectroscopy confirmed that radiative recombination in the fabricated sample dominated in the whole temperature range up to $300 \mathrm{~K}$. This leads to record weak temperature-induced quenching of the $\mathrm{QW}$ emission intensity, which at $300 \mathrm{~K}$ does not exceed $20 \%$ of the low-temperature value.

Key words: AlGaN, quantum wells, excitons, molecular beam epitaxy

\section{INTRODUCTION}

AlGaN-based quantum well (QW) heterostructures are of great importance for manufacturing high-efficiency emitters for the mid-ultraviolet (mid-UV) spectral range $(\lambda<300 \mathrm{~nm})$. This is an essential segment for photonics, where low-efficiency, bulky, and toxic Hg-containing vacuum bulbs are still mainly used for numerous ecological, medical, and military/security applications. ${ }^{1}$ Application of AlGaN QWs is, however, hampered by the lack of commercially available latticematched substrates. As a result, such structures suffer from enhanced density of threading dislocations (more than $10^{9} \mathrm{~cm}^{-2}$ for growth on $c$-sapphire), and in spite of the high internal quantum efficiency of emission at low temperatures,

(Received July 21, 2016; accepted October 22, 2016;

published online November 8, 2016) achievement of reasonably high efficiency at $300 \mathrm{~K}$ relies on realization of an efficient localization potential to prevent charge carrier transport to nonradiative recombination centers. ${ }^{2-4}$ This localization implies both strong confinement by the one-dimensional QW potential, preventing vertical transport of carriers along the growth axis, and a random localization potential in lateral dimensions, which hampers their migration in the plane of the QW. Enhancement of the vertical confinement essentially depends on the proper choice of the basic QW parameters, such as the width and composition of the well and barrier layers, whereas the random in-plane potential originates mainly from well-barrier interface roughness and alloy disorder. It benefits from use of narrower wells and implementation of specific technological regimes promoting emergence of enhanced compositional modulation. ${ }^{5-7}$ 
The physics of III-nitride QWs with wurtzite structure differs from the physics of longer and better studied zincblende QWs in two main aspects: First, the confining potential of wurtzite QWs always deviates from the rectangular one due to the built-in electric field induced by spontaneous and piezoelectric polarizations. ${ }^{8,9}$ Therefore, the activation energy required for thermal excitation of confined charge carriers into the $\mathrm{QW}$ barriers is a complicated function of the composition and thickness of all the constituent layers of the heterostructure, as well as their elastic strain. Second, the calculation of the valence-band structure of a wurtzite semiconductor is complicated by the necessity to take into account closely spaced heavy-hole, light-hole, and crystal-field split-off bands simultaneously. ${ }^{10-12}$ This is especially important for $\mathrm{Al}_{x}$ $\mathrm{Ga}_{1-x} \mathrm{~N}$ alloys with $x>0.5$, emitting in the mid-UV spectral range, since only these compounds grown coherently on AlN buffers or substrates exhibit inversion of valence-band ordering. ${ }^{13,14}$ Along with the complexity of state-of-the-art growth technology for wurtzite heterostructures, these two issues hinder systematic optimization of mid-UV-emitting QW structures, which to the best of our knowledge has not been reported to date.

We present herein optimization of the design of an $\mathrm{Al}_{x} \mathrm{Ga}_{1-x} \mathrm{~N} / \mathrm{Al}_{y} \mathrm{Ga}_{1-y} \mathrm{~N} \mathrm{QW}$ heterostructure emitting mid-UV light with transverse electric (TE) polarization, aiming at achievement of the maximum possible internal quantum efficiency at $300 \mathrm{~K}$. The performed band and excitonic calculations allow us to define the optimum width and composition for the QW, whereas careful development of growth of such structures using molecular beam epitaxy (MBE) led to fabrication of strongly disordered "digital" QWs. As a result, we present a QW heterostructure emitting below $300 \mathrm{~nm}$ whose photoexcited carrier lifetime is defined by radiative recombination throughout the whole temperature range between $5 \mathrm{~K}$ and $300 \mathrm{~K}$ and whose internal quantum efficiency is only weakly temperature dependent.

\section{THEORETICAL OPTIMIZATION AND STRUCTURE DESIGN}

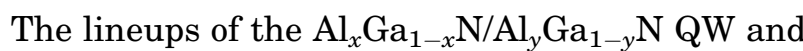
involved effective masses of charge carriers were defined within a six-band $k \cdot p$ model,${ }^{10}$ taking into account elastic strain induced by pseudomorphic growth on an AlN buffer. All low-temperature material parameters involved were taken from Ref. 15, except for the GaN and AlN deformation potentials, which were taken from Refs. 16 and 17. Growth on an AlN buffer has the benefit of introducing the largest possible strain in both the $\mathrm{Al}_{x} \mathrm{Ga}_{1-x} \mathrm{~N} \mathrm{QW}$ and $\mathrm{Al}_{y} \mathrm{Ga}_{1-y} \mathrm{~N}$ barriers. As a result, the topmost valence band in the $\mathrm{Al}_{x} \mathrm{Ga}_{1-x} \mathrm{~N}$ alloy is the heavy-hole band up to the limiting Al content of $x \approx 0.6$, allowing efficient TE-polarized emission from surface-emitting devices down to wavelength of $\sim 240 \mathrm{~nm} .{ }^{14}$ For $x>0.6$, the topmost valence band is the crystal-field split-off band and the respective interband transition is transverse magnetic (TM) polarized. The QW potential profile was approximated using the model of a homogeneous electric field, taking into account both piezo- and spontaneous polarizations. Single-particle envelope wavefunctions and eigenfrequencies were obtained by using a transfer-matrix method, whereas QW excitonic parameters were calculated variationally using a two-parameter probe function. ${ }^{18,19}$ To avoid cumbersome multiband excitonic calculations, we employed one-band envelope functions in the variational procedure, neglecting mixing in the ground QW exciton of hole states from various valence bands. This choice is justified by the fact that, in the considered QWs with TE-polarized emission, the ground excitonic state involves heavy holes, which do not couple at $k_{\mathrm{t}}=0$ with either the light-hole or crystal-field split-off bands. ${ }^{11,20}$

An important parameter that affects the internal quantum efficiency is the activation energy required for thermal excitation of photoexcited carriers from the QW to the barriers. For the most attractive accessible wavelengths for TE-polarized emission between $300 \mathrm{~nm}$ and $240 \mathrm{~nm}$, the $\mathrm{Al}$ mole fraction in the barriers should be above the band-crossing point $(y>0.6)$, whereas the $\mathrm{Al}$ mole fraction in the $\mathrm{QW}$ should be below it $(x<0.6)$. Therefore, the hole activation energy is defined as the gap between the ground heavy-hole QW energy level and the top of the split-off valence band in the lowest hole barrier of the asymmetric QW. The electron activation energy is defined as the gap between the ground electron QW energy level and the bottom of the conduction band in the opposite barrier layer.

Figure 1 shows the activation energies calculated for electrons (Fig. 1a) and heavy holes (Fig. 1b) as functions of the QW width for several selected combinations of QW and barrier alloy compositions: $x=0.4$ and $y=0.6,0.8$, and 1.0. The activation energies naturally increase with both $y$ and the $\mathrm{QW}$ width. However, the dependence on the $\mathrm{QW}$ width rapidly saturates at approximately $7 \AA$ to $9 \AA$ for heavy holes and approximately $12 \AA$ to $15 \AA$ for electrons. These values correspond to sinking of the energy levels in a triangular part of the QW profile so that the activation energies in the thicker QWs are determined by the value of the intrinsic electric field rather than by the $\mathrm{QW}$ width. Increasing the QW width above these values is useless, since it does not extend the activation energies but decreases both the radiative recombination rate and exciton binding energy.

The respective exciton resonance wavelength and exciton binding energy are plotted in Fig. $2 \mathrm{a}$ and $\mathrm{b}$ versus the $\mathrm{QW}$ width. Near the threshold values of the QW width ( $12 \AA$ to $15 \AA$ ), the exciton position is practically independent of the barrier composition, being defined only by the $\mathrm{Al}$ content in the $\mathrm{QW}$. This 


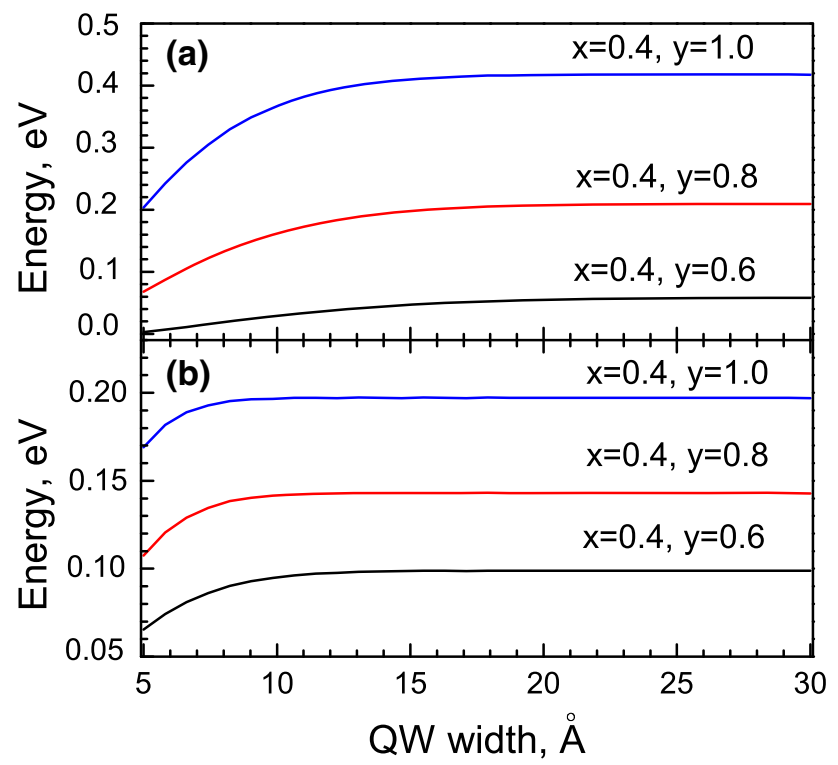

Fig. 1. Activation energy of electrons (a) and heavy holes (b) calculated as functions of QW width in $\mathrm{Al}_{x} \mathrm{Ga}_{1-x} \mathrm{~N} / \mathrm{Al}_{y} \mathrm{Ga}_{1-y} \mathrm{~N}$ QWs for various combinations of $x$ and $y$.

behavior simply originates from the fact that the confinement energies increase with the barrier height in the rectangular $\mathrm{QW}$, whereas in the triangular QW the quantum confined Stark effect decreases the transition energy progressively with increase of the $\mathrm{Al}$ content in the barriers, following enhancement of the intrinsic electric field induced mainly by the difference in the spontaneous polarizations of the QW and barriers. One can see in Fig. $2 \mathrm{~b}$ that the exciton binding energy near the threshold width is large enough (more than $40 \mathrm{meV}$ ), provided the $\mathrm{Al}$ content in the barriers is sufficiently large $(y>0.6)$. Strong Coulomb attraction between the electron and hole facilitates thermal activation of an exciton as a whole rather than separate excitation of unipolar electrons and holes. Under these conditions, the exciton activation energy can be estimated as the sum of the electron and hole activation energies, as previously shown for narrow GaN/AlGaN QWs. ${ }^{21}$

Figure 3 summarizes the exciton activation energies (Fig. 3a) and exciton resonance wavelengths (Fig. 3b) for the chosen QW width of $14 \AA$ and extended ranges of $\mathrm{QW}$ and barrier compositions. Horizontal dashed lines indicate target values of exciton activation energy and resonance wavelength. For the activation energy, we adopt a target value of $0.3 \mathrm{eV}$. When substituted into the conventional expression for the thermally activated photoluminescence (PL) intensity with the preexponential factor taken from Ref. 21, this value leads to radiative efficiency above $80 \%$ at $300 \mathrm{~K}$. The target value for the low-temperature exciton resonance wavelength is $270 \mathrm{~nm}$. Bearing in mind a Stokes shift between the absorption and emission lines as well as a temperature-induced red-shift,

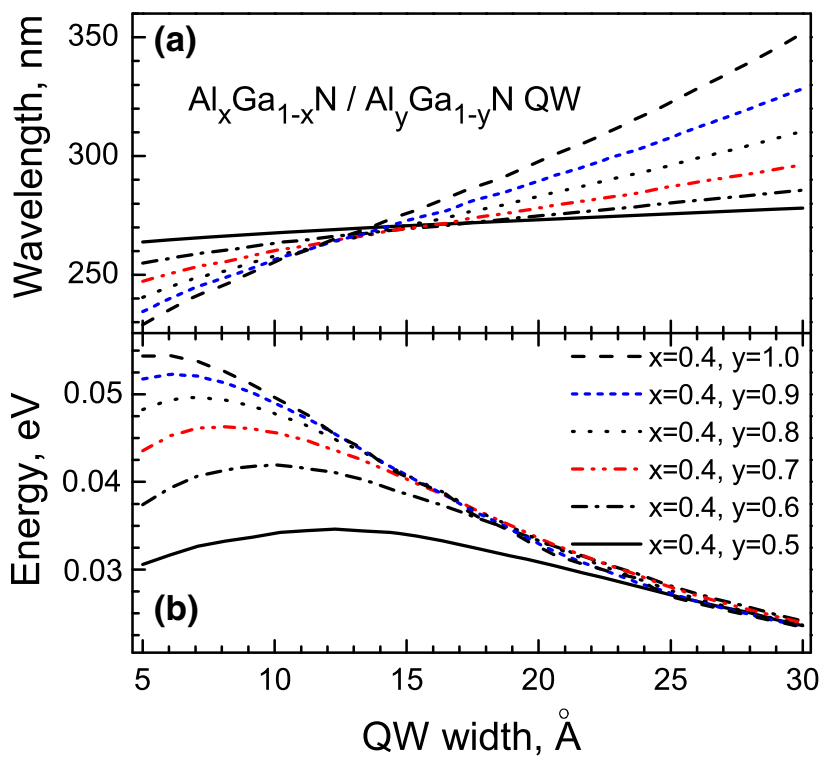

Fig. 2. Exciton resonance wavelength (a) and exciton binding energy (b) calculated as functions of $\mathrm{QW}$ width for $\mathrm{Al}_{x} \mathrm{Ga}_{1-x} \mathrm{~N} / \mathrm{Al}_{y}$ $\mathrm{Ga}_{1-y} \mathrm{~N}$ QWs for various combinations of $x$ and $y$.

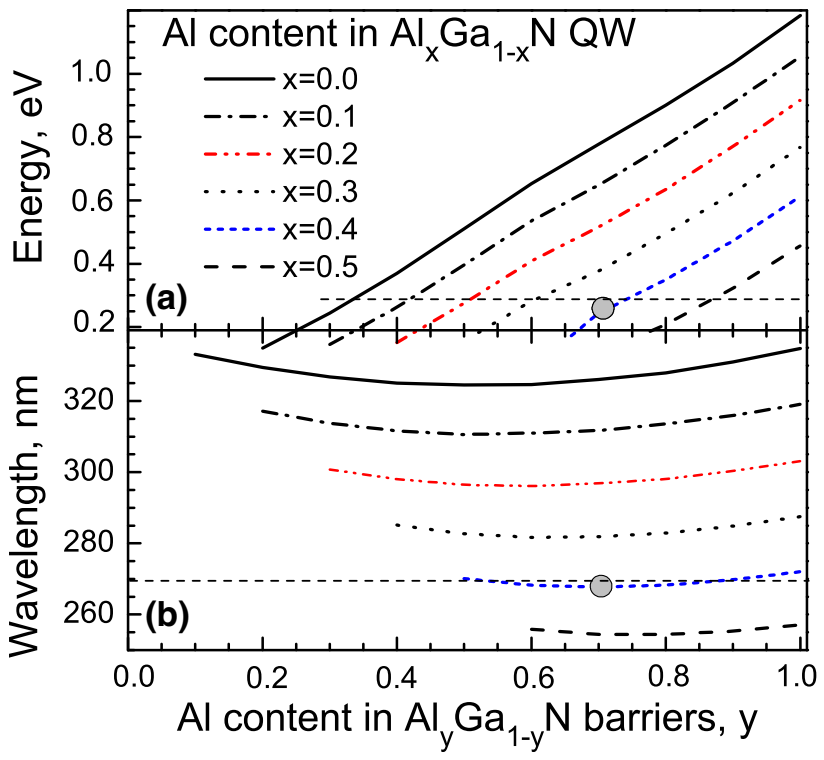

Fig. 3. Activation energy of electron-hole pair (a) and exciton resonance wavelength (b) calculated for a $14-\mathrm{A}$-thick $\mathrm{Al}_{x} \mathrm{Ga}_{1-x} \mathrm{~N} / \mathrm{Al}_{y}$ $\mathrm{Ga}_{1-y} \mathrm{~N}$ QW for selected values of $x$ versus $y$. Horizontal dashed lines highlight designed values of activation energy $(0.3 \mathrm{eV})$ and exciton resonance wavelength $(270 \mathrm{~nm})$. Gray circles mark positions relevant to the fabricated $\mathrm{QW}$ structure.

this value should result in a room-temperature emission line just below $300 \mathrm{~nm}$. One can see in Fig. 3 that, for the chosen QW width, these values can be achieved for $x>0.4$ and $y>0.7$. Minimizing the $\mathrm{Al}$ content in the barriers and pursuing the $\mathrm{TE}$ emission polarization, we fixed the following set of QW parameters: $x=0.4, y=0.7$, and QW width of $14 \AA$. 


\section{SAMPLE GROWTH AND EXPERIMENTAL PROCEDURES}

A structure with a single $14-\AA$-thick $\mathrm{Al}_{0.4} \mathrm{Ga}_{0.6} \mathrm{~N} /$ $\mathrm{Al}_{0.7} \mathrm{Ga}_{0.3} \mathrm{~N} \quad \mathrm{QW}$ was grown by plasma-assisted molecular beam epitaxy on a $c$-sapphire substrate using a $2-\mu \mathrm{m}$-thick AlN buffer with thin GaN insertions as a dislocation filter. The typical density of threading dislocations in thus-grown structures was previously estimated as $\sim 10^{9} \mathrm{~cm}^{-2} \cdot 22-24$ The active region of the structure was grown pseudomorphically under metal-rich conditions. It consisted of a 32-nm-thick $\mathrm{Al}_{0.7} \mathrm{Ga}_{0.3} \mathrm{~N}$ barrier, a 1.5nm-thick $\mathrm{Al}_{0.4} \mathrm{Ga}_{0.7} \mathrm{~N}$ QW, a 65-nm-thick $\mathrm{Al}_{0.7 \rightarrow 1}$ $\mathrm{Ga}_{0.3 \rightarrow 0} \mathrm{~N}$ graded barrier layer, and $10-\mathrm{nm}$-thick AlN capping. During the growth of the barrier layers, the substrate was rotated sufficiently fast (one rotation per $3 \mathrm{~s}$ ) to ensure formation of homogeneous alloy without pronounced spontaneous compositional modulation. ${ }^{25}$

The QW was formed by the submonolayer digital alloying (SDA) technique ${ }^{22,26}$ as a superlattice of $10 \times \mathrm{GaN} / \mathrm{Al}_{0.7} \mathrm{Ga}_{0.3} \mathrm{~N}$, with growth of $\mathrm{GaN}$ layers achieved by briefly shutting off the $\mathrm{Al}$ source. Nominal thicknesses of the constituent GaN and $\mathrm{Al}_{y} \mathrm{Ga}_{1-y} \mathrm{~N}$ layers were selected in such a way that the average QW thickness and composition matched the intended parameter values. The $\mathrm{QW}$ growth was performed with a reduced rate of substrate rotation ( 1 rotation per $7 \mathrm{~s}$ ). It was previously shown that the SDA technique results in strong compositional modulation inside the QW layer, which should facilitate implementation of a localization potential preventing in-plane transport of photoexcited carriers. ${ }^{27}$ The surface morphology was controlled in situ by means of reflection high-energy electron diffraction and after growth by high-angle annular darkfield scanning transmission electron microscopy (HAADF STEM).

The stress evolution in the heterostructure was monitored in real time during growth using an original multibeam optical stress sensor (MOSS) intended for standard measurements of substrate curvature $(k)$, which is related to film stress $(\sigma)$ through Stoney's equation $k=6 \sigma h_{\mathrm{f}} /\left(M_{\mathrm{s}} h_{\mathrm{s}}^{2}\right)$, where $h_{\mathrm{f}(\mathrm{s})}$ is the film (substrate) thickness and $M_{\mathrm{s}}$ is the substrate biaxial modulus (602 GPa for $\left.c-\mathrm{Al}_{2} \mathrm{O}_{3}\right)$. A relatively high measurement frequency (up to $5 \mathrm{~Hz}$ ) was used in our experiments to improve the temporal resolution of the MOSS technique.

Photoluminescence (PL) spectra were measured using the third harmonic of a titanium-doped sapphire laser $(\sim 255 \mathrm{~nm})$ generating 150-fs-long pulses with frequency of $76 \mathrm{MHz}$. This wavelength corresponds to a photon energy below the bandgap of the $\mathrm{Al}_{0.7} \mathrm{Ga}_{0.3} \mathrm{~N}$ barriers, providing direct excitation of charge carriers (or excitons) within the $\mathrm{Al}_{0.4} \mathrm{Ga}_{0.6} \mathrm{~N}$ QW. Time-integrated PL spectra were measured using a cooled charge-coupled device (CCD) camera, whereas time-resolved PL was detected using either a Hamamatsu streak camera (ultimate resolution $\sim 15 \mathrm{ps)}$ or a fast

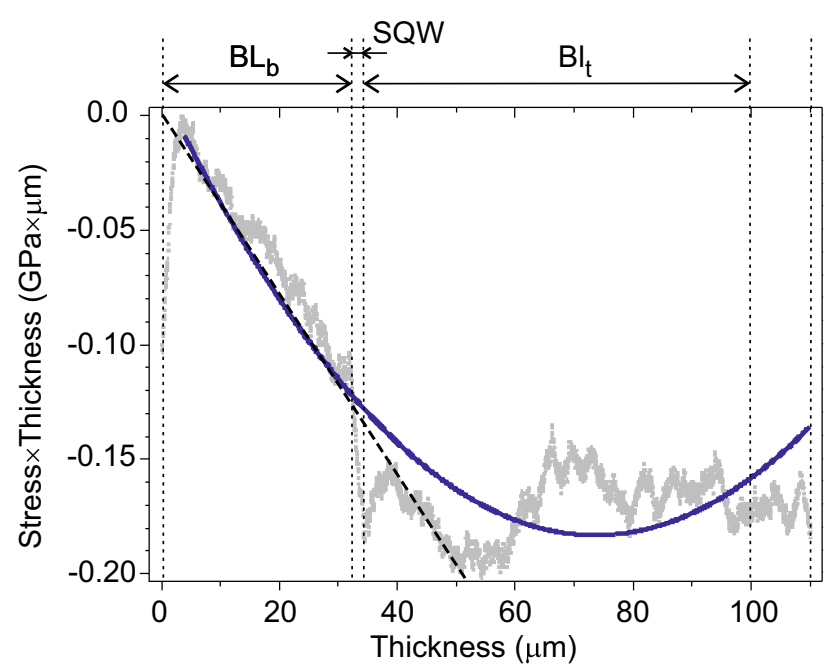

Fig. 4. Real-time MOSS data obtained during growth of the $\mathrm{Al}_{0.7}$ $\mathrm{Ga}_{0.3} \mathrm{~N}$ barrier layer before insertion of the 1.4-nm-thick $\mathrm{Al}_{0.4} \mathrm{Ga}_{0.6} \mathrm{~N}$ SQW (during the first $32 \mathrm{~nm}$ of $\mathrm{BL}$ ) and the $\mathrm{Al}_{x} \mathrm{Ga}_{1-x} \mathrm{~N}$ ( $x=0.7$ to 1 ) graded layer after the SQW (grey dots). The continuous line is a polynomial approximation to the experimental data, while the dashed line shows the calculated dependence of [stress $\times$ thickness] on the thickness for coherent growth of an $\mathrm{Al}_{0.7} \mathrm{Ga}_{0.3} \mathrm{~N}$ layer on an $\mathrm{AIN}$ buffer.

photomultiplier operating in time-correlated photon-counting mode (resolution $\sim 130 \mathrm{ps}$ ). The latter technique is especially useful for evaluation of relatively long PL decay times exceeding a few nanoseconds.

\section{EXPERIMENTAL RESULTS AND DISCUSSION}

Figure 4 shows the evolution of the stress $\times$ thickness parameter during growth of the barrier layer (BL), containing the single QW (SQW), above the thick AlN buffer layer. The constant slope at any point on the graph before insertion of the SQW (part $\mathrm{BL}_{\mathrm{b}}$ in Fig. 4) represents an instantaneous stress corresponding to an estimated value (dashed line) calculated using biaxial modulus values of $M_{\mathrm{GaN}}=478 \mathrm{GPa}$ and $M_{\mathrm{AlN}}=510 \mathrm{GPa},{ }^{28}$ as well as the Vegard approximation for the ternary alloy. The change of sign of the stress after the SQW insertion corresponds to the transition to the growth of $\mathrm{Al}_{x} \mathrm{Ga}_{1-x} \mathrm{~N}$ layer with a positive linear gradient of the Al content from $x=0.7$ to 1 (part $\mathrm{BL}_{\mathrm{t}}$ in Fig. 4). Thus, these MOSS measurement data reveal the absence of any noticeable strain relief during the growth of the $\mathrm{Al}_{0.7} \mathrm{Ga}_{0.3} \mathrm{~N}$ barrier layer before and just after insertion of the SQW.

Figure 5 shows a cross-sectional HAADF STEM image of the structure, taken close to the position of the SQW. The image reveals the distribution of the chemical composition within the AlGaN alloys, resolved on the scale of atomic layers, so that brighter contrast in the image corresponds to higher Ga content. The SQW manifests itself as enhanced Ga content, distributed inhomogeneously within a 


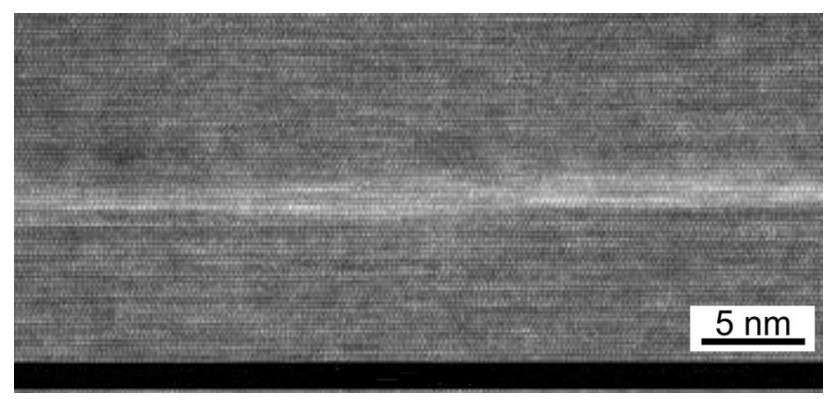

Fig. 5. Cross-sectional HAADF STEM image of the fabricated heterostructure in the vicinity of the $\mathrm{Al}_{0.4} \mathrm{Ga}_{0.6} \mathrm{~N} / \mathrm{Al}_{0.7} \mathrm{Ga}_{0.3} \mathrm{~N} \mathrm{QW}$ with average width of $1.4 \mathrm{~nm}$ and strong compositional modulation. Brighter contrast corresponds to higher Ga content in AIGaN alloy.

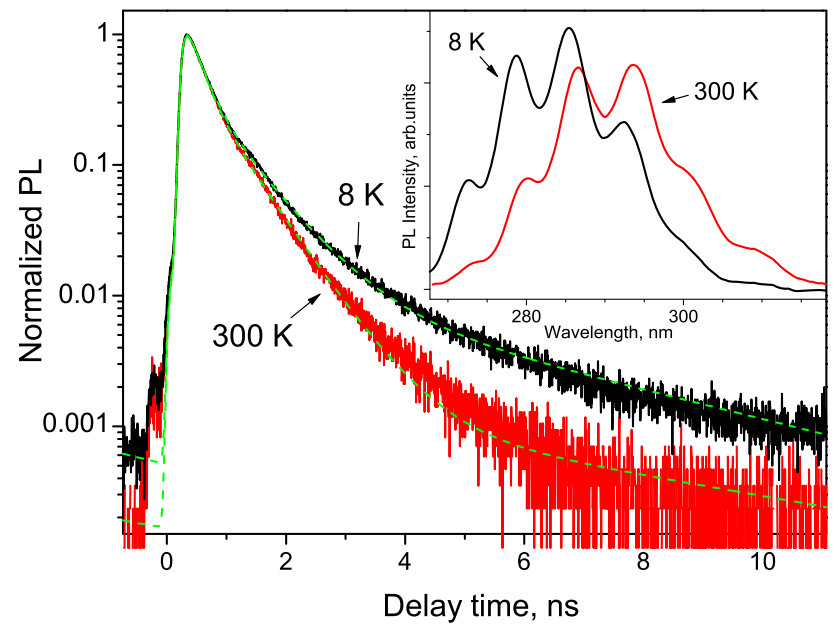

Fig. 6. Normalized PL decay curves measured in $\mathrm{Al}_{0.4} \mathrm{Ga}_{0.6} \mathrm{~N} / \mathrm{Al} \mathrm{I}_{0.7}$ $\mathrm{Ga}_{0.3} \mathrm{~N} \mathrm{QW}$ at $8 \mathrm{~K}$ and $300 \mathrm{~K}$ at wavelengths corresponding to the center of the emission line. Dashed lines show best fits to the data. The inset shows time-integrated spectra measured at $8 \mathrm{~K}$ and $300 \mathrm{~K}$.

stripe having average thickness close to the intended value of $14 \AA$. The observed thickness/composition fluctuations are expected to result in strong potential fluctuations preventing transport of strongly localized excitons in the plane of the QW.

The inset in Fig. 6 shows time-integrated spectra measured from the fabricated sample at $8 \mathrm{~K}$ and $300 \mathrm{~K}$. The spectra display a single wide PL line attributed to emission of localized excitons in the SQW. The spectrum is modulated by a periodic structure attributed to light interference in the thick buffer layers. ${ }^{25}$ Temperature increase causes a red-shift of the peak, but even at $300 \mathrm{~K}$, most of the line is below $300 \mathrm{~nm}$, in agreement with the target emission wavelength. Remarkably, the temperature-induced decrease in the PL intensity at $300 \mathrm{~K}$ does not exceed $20 \%$ of the low-temperature value, in agreement with the optimization performed for the sample design and the growth procedure.

Figure 6 shows the normalized PL decay curves measured at the center of the emission line at $8 \mathrm{~K}$ and $300 \mathrm{~K}$. At $300 \mathrm{~K}$, the PL decay curve can be fit by a sum of two decaying exponents with decay time constants of $\sim 210 \mathrm{ps}$ and $\sim 650 \mathrm{ps}$. Decrease of the temperature to $8 \mathrm{~K}$ does not modify these time constants but rather leads to the appearance of an additional weak slowly decaying component with time constant as long as $5 \mathrm{~ns}$ to $6 \mathrm{~ns}$. These data indicate the strongly inhomogeneous nature of the PL line involving emission of excitons localized at various distances from threading dislocations and other defects. The observed temperature independence of the dominant time constants indicates a negligible role for nonradiative recombination across the whole temperature range up to $300 \mathrm{~K}$.

\section{CONCLUSIONS}

Based on theoretical consideration of exciton activation and binding energies in an $\mathrm{Al}_{x} \mathrm{Ga}_{1-x} \mathrm{~N} /$ $\mathrm{Al}_{y} \mathrm{Ga}_{1-y} \mathrm{~N} \mathrm{QW}$ emitting below $300 \mathrm{~nm}$, we elucidate optimal ranges of $\mathrm{QW}$ width and composition, leading to maximum internal quantum efficiency at $300 \mathrm{~K}$. A fabricated sample with such an optimized SQW showed record weak PL quenching with temperature, as low as $\sim 20 \%$ for temperature variation between $5 \mathrm{~K}$ and $300 \mathrm{~K}$. These data evidence efficient spatial localization of photoexcited carriers due to both vertical confinement within the $\mathrm{QW}$ and lateral localization in the $\mathrm{QW}$ plane. Vertical confinement is strongly favored by the proper choice of the optimal QW width and composition, while the efficiency of lateral localization essentially relies on the use of the SDA technique for QW growth. Thus, such optimized grown heterostructures are very promising for development of efficient mid-UV light-emitting devices.

\section{ACKNOWLEDGEMENTS}

This work was partly supported by the Russian Foundation for Basic Research (Grant No. 15-0205206a).

\section{REFERENCES}

1. M. Kneissl and J. Rass, III-Nitride Ultraviolet Emitters: Technology and Applications. Springer Series in Materials Science, Vol. 227 (Springer International Publ., New York, 2016).

2. S.F. Chichibu, A. Uedono, T. Onuma, B.A. Haskell, A. Chakraborty, T. Koyama, P.T. Fini, S. Keller, S.P. DenBaars, J.S. Speck, U.K. Mishra, S. Nakamura, S. Yamaguchi, S. Kamiyama, H. Amano, I. Akasaki, J. Han, and T. Sota, Nat. Mater. 5, 810 (2006).

3. I. Friel, C. Thomidis, and T.D. Moustakas, Appl. Phys. Lett. 85, 3068 (2004).

4. M. Shatalov, J. Yang, W. Sun, R. Kennedy, R. Gaska, K. Liu, M. Shur, and G. Tamulaitis, J. Appl. Phys. 105, 073103 (2009).

5. D. Korakakis, K.F. Ludwig Jr, and T.D. Moustakas, Appl. Phys. Lett. 71, 72 (1997).

6. M. Gao, S.T. Bradley, Y. Cao, D. Jena, Y. Lin, S.A. Ringel, J. Hwang, W.J. Schaff, and L.J. Brillson, J. Appl. Phys. $100,103512(2006)$.

7. Y. Iwata, T. Oto, D. Gachet, R.G. Banal, M. Funato, and Y. Kawakami, J. Appl. Phys. 117, 115702 (2015).

8. A. Bykhovski, B. Gelmont, and M. Shur, J. Appl. Phys. 74, 6734 (1993). 
9. F. Bernardini, V. Fiorentini, and D. Vanderbilt, Phys. Rev. B 56, R10024 (1997).

10. G.I. Bir and G.E. Pikus, Symmetry and Strain-Induced Effects in Semiconductors (New York: Wiley, 1974).

11. S.L. Chuang and C.S. Chang, Phys. Rev. B 54, 2491 (1996).

12. YuM Sirenko, J.-B. Jeon, K.W. Kim, M.A. Littlejohn, and M.A. Stroscio, Phys. Rev. B 53, 1997 (1996).

13. H. Kawanishi, M. Senuma, M. Yamamoto, E. Niikura, and T. Nukui, Appl. Phys. Lett. 89, 081121 (2006).

14. J.E. Northrup, C.L. Chua, Z. Yang, T. Wunderer, M. Kneissl, N.M. Johnson, and T. Kolbe, Appl. Phys. Lett. 100, 021101 (2012).

15. I. Vurgaftman and J.R. Meyer, J. Appl. Phys. 94, 3675 (2003).

16. R. Ishii, A. Kaneta, M. Funato, Y. Kawakami, and A.A. Yamaguchi, Phys. Rev. B 81, 155202 (2010).

17. R. Ishii, A. Kaneta, M. Funato, and Y. Kawakami, Phys. Rev. B 87, 235201 (2013).

18. Y. Shinozuka and M. Matsuura, Phys. Rev. B 28, 4878 (1983).

19. A.A. Toropov, E.A. Shevchenko, T.V. Shubina, V.N. Jmerik, D.V. Nechaev, M.A. Yagovkina, A.A. Sitnikova, S.V. Ivanov, G. Pozina, J.P. Bergman, and B. Monemar, J. Appl. Phys. 114, 124306 (2013).

20. H. Lu, T. Yu, G. Yuan, C. Jia, G. Chen, and G. Zhang, Opt. Express 20, 27384 (2012).
21. N. Grandjean, J. Massies, I. Grzegory, and S. Porowski, Semicond. Sci. Technol. 16, 358 (2001).

22. V.N. Jmerik, A.M. Mizerov, A.A. Sitnikova, P.S. Kop'ev, S.V. Ivanov, E.V. Lutsenko, N.P. Tarasuk, N.V. Rzheutskii, and G.P. Yablonskii, Appl. Phys. Lett. 96, 141112 (2010).

23. V.N. Jmerik, E.V. Lutsenko, and S.V. Ivanov, Phys. Status Solidi A 210, 439 (2013).

24. S.V. Ivanov, D.V. Nechaev, A.A. Sitnikova, V.V. Ratnikov, M.A. Yagovkina, N.V. Rzheutskii, E.V. Lutsenko, and V.N. Jmerik, Semicond. Sci. Technol. 29, 084008 (2014).

25. A.A. Toropov, E.A. Shevchenko, T.V. Shubina, V.N. Jmerik, D.V. Nechaev, G. Pozina, P. Bergman, B. Monemar, S. Rouvimov, and S.V. Ivanov, Phys. Stat. Sol. C 13, 232 (2016).

26. X. Rong, X. Wang, S.V. Ivanov, X. Jiang, G. Chen, P. Wang, W. Wang, C. He, T. Wang, T. Schulz, M. Albrecht, V.N. Jmerik, A.A. Toropov, V.V. Ratnikov, V.I. Kozlovsky, V.P. Martovitsky, P. Jin, F. Xu, X. Yang, Z. Qin, W. Ge, J. Shi, and B. Shen, Adv. Mater. 28, 7978 (2016).

27. V.N. Jmerik, D.V. Nechaev, S. Rouvimov, V.V. Ratnikov, P.S. Kop'ev, M.V. Rzheutski, E.V. Lutsenko, G.P. Yablonskii, M. Aljohenii, A. Aljerwii, A. Alyamani, and S.V. Ivanov, J. Mater. Res. 30, 2871 (2015).

28. A. Polian, M. Grimsditch, and I.G. Grzegory, J. Appl. Phys. 79, 3343 (1996). 